\title{
Graft-versus-host disease after deceased donor kidney transplantation
}

\author{
Hojong Park ${ }^{1}$, Sang Jun Park ${ }^{1}$, Hong Rae Cho ${ }^{1}$, Kyung Sun Park², Jongha Park², Kyung Don Yoo ${ }^{2}$, Jong Soo Lee ${ }^{2}$ \\ ${ }^{1}$ Department of Surgery, Ulsan University Hospital, Ulsan, Korea \\ ${ }^{2}$ Division of Nephrology, Department of Internal Medicine, Ulsan University Hospital, Ulsan, Korea
}

Background: Graft-versus-host disease (GVHD) is a rare complication after solid organ transplantation. We report a case of GVHD in recipient after deceased donor kidney transplantation.

Methods: The recipient was a 49-year-old female patient with end-stage renal disease due to hypertension and chronic glomerulonephritis. She was on hemodialysis for 11 years. The donor was a 45-year-old male. The deceased donor kidney transplantation was performed on February 22, 2020. Two months postoperatively she developed anorexia and severe epigastric pain. An esophagogastroduodenoscopy was performed and revealed multiple shallow ulcerative lesion were noted at fundus, high body, mid-body. Since it was in the early stage of transplantation, it was considered as a steroid induced ulcer. After taking ulcer medication, the symptoms did not improved even after 1 week. The biopsy was performed after retesting the endoscope. Biopsy findings were consistent with GVHD. When the patient was examined closely and in detail, skin lesions on trunk and thigh were found. Skin biopsy was performed for differential diagnosis, and favored GVHD. Aggressive immunosuppressive therapy was instituted with a good response.

Results: The anorexia and epigastric pain resolved, and she was discharged on hospital day 38. Three months later, there had been no recurrence of anorexia, epigastric pain. A repeat endoscopy revealed complete resolution of the initial endoscopic abnormalities, and there were no GVHD findings on biopsy. Eight months after transplantation, the patient was asymptomatic with normal graft function.

Conclusions: GVHD after kidney transplantation is rare. In particular, in the case of gastric GVHD, it may be difficult to discriminate due to immunosuppressants such as steroids at the early stage of transplantation. If symptoms do not improve even after ulcer treatment, it is important to actively perform endoscopy and biopsy for early diagnosis and treatment.

Corresponding author: Hojong Park

E-mail:0732840@uuh.ulsan.kr

(C) The Korean Society for Transplantation

This is an Open Access article distributed under the terms of the Creative Commons Attribution Non-Commercial License (http://creativecommons.org/licenses/by-nc/4.0/) which permits unrestricted non-commercial use, distribution, and reproduction in any medium, provided the original work is properly cited. 